\begin{tabular}{|c|l|}
\hline Title & Determination of the transphosphorylation sites of Jak2 kinase. \\
\hline Author(s) & Matsuda, Tadashi; Feng, Jian; Witthuhn, Bruce A.; Sekine, Y uichi; Ihle, James N. \\
\hline Citation & $\begin{array}{l}\text { Biochemical and Biophysical Research Communications, 325(2), 586-594 } \\
\text { https://doi.org/10.1016/.bbrc.2004.10.071 }\end{array}$ \\
\hline Issue Date & 2004 12-10 \\
\hline Doc URL & http://hdl.handle.net/2115/28122 \\
\hline Type & article (author version) \\
\hline File Information & NNRC325-2.pdf \\
\hline
\end{tabular}

Instructions for use 


\title{
Determination of the Transphosphorylation Sites of Jak2 Kinase
}

\author{
Tadashi Matsuda $^{3}$, Jian Feng ${ }^{4}$, Bruce A Witthuhn ${ }^{2}$, Yuichi Sekine ${ }^{3}$ \\ and James N Ihle ${ }^{1}$
}

From the ${ }^{1}$ Department of Biochemistry, St Jude Research Hospital, ${ }^{2}$ The Department of Biochemistry, Molecular Biology and Children’s Biophysics, University of Minnesota, St Paul, MN, ${ }^{3}$ Department of Immunology, Graduate School of Pharmaceutical Sciences, Hokkaido University, Sapporo, Japan, and the ${ }^{4}$ Department of Physiology and Biophysics, State University of New York at Buffalo, Buffalo, NY

Keywords: Jak, transphosphorylation

Corresponding author: Tadashi Matsuda, ${ }^{3}$ Department of Immunology, Graduate School of Pharmaceutical Sciences, Hokkaido University, Kita-Ku Kita 12 Nishi 6, Sapporo 060-0812, Japan, TEL: 81-11-706-3243, FAX: 81-11-706-4990, Email: tmatsuda@pharm.hokudai.ac.jp

Running title: Transphosphorylation sites of Jak2 


\begin{abstract}
Janus kinases are the key enzymes involved in the initial transmission of signals in response to type I and II cytokines. Activation of the signal begins with the transphosphorylation of Jak kinases. Substrates that give rise to downstream events are recruited to the receptor complex in part by interactions with phosphorylated tyrosines. The identity of many of the phosphotyrosines responsible for recruitment has been elucidated as being receptor-based tyrosines. The ability of Jaks to recruit substrates through their own phosphotyrosines has been demonstrated for tyrosines in the kinase activation loop. Recent studies demonstrate that other tyrosines have implications in regulatory roles of Jak kinase activity. In this study, baculovirusproduced Jak2 was utilized to demonstrate that transphosphorylation of Jak kinases occurs on multiple residues throughout the protein. We demonstrate that among the tyrosines phosphorylated, those in the kinase domain occur as expected, but many other sites are also phosphorylated including $\mathrm{Y}^{221}$ and $\mathrm{Y}^{570}$. The tyrosines conserved in the Jak family are the object of this study, although many of them are phosphorylated, many are not. This result suggests that conservation of tyrosines is perhaps as important in maintaining structure of the Jak family. Additionally, non-Jak family conserved tyrosines are phosphorylated suggesting that the individual Jaks ability to phosphorylated specific tyrosines may influence signals emitting from activated Jaks.
\end{abstract}




\section{Introduction}

The Janus protein tyrosine kinase (Jaks) have been most consistently implicated as a family of protein tyrosine kinases activated in cytokine signaling(1). Genetic and mutational analysis has established Jak kinases to be essential to type I and II cytokine receptors lacking protein tyrosine kinase domains (2). The Jak family of kinases includes four mammalian members, Jak1, Jak2, Jak3 and Tyk2 (3) and to date a single member found in drosophila, hopscotch (4). Besides a tyrosine kinase domain (JH1), the Jak family share six additional homology units defined as Jak homology domains (JH2-JH7) (Fig 1A). The JH2 domain, which is immediately amino terminal of the tyrosine kinase domain (JH1), has sequence similarity to a kinase domain, but lacks a consensus autophosphorylation motif and a functional ATP binding domain. Mutational and deletion analyses have demonstrated that this region is not essential for Jak2 tyrosine kinase activity, but is implicated in regulating kinase activity (5-8). A point mutation within the JH2 domain of Hopscotch renders the enzyme constitutively active (9). However, no similar activating mutation has been reported in mammals. Conversely, loss-of-function mutations have been reported in the JH2 domain of Jak3, which causes SCIDS symptoms (8). In addition, poor IFN- $\gamma$ response has been linked to mutations in the JH2 domain of Tyk2 $(10,11)$.

Contained within the JH3 domains is a putative SH2 motif. Studies preformed on Jak3’s putative SH2 domain have demonstrated association with phosphorylated proteins, but no physiological significance has been established (12). Mutational analysis of the

conserved Arg residue in the SH2 domain of Jak2 has no consequences on IFN $\gamma$ signaling (13). The JH7 and JH6 domains have within them a FERM domain (14-17) which has Jak2 transphosphorylation 
been shown in band 4.1 protein from erythrocytes to be necessary for bridging the actin cytoskeleton to the membrane $(18,19)$. The functional significance of the FERM domain in JAK kinases is relatively unexplored, but the existence of a FERM domain in the region of Jaks responsible for receptor association is consistent with the proximity of Jak kinases to the plasma membrane (20-22).

Upon ligand binding on cytokine receptors, a series of events occur, which results in specific responses characteristic of a particular cytokine receptor. The most immediate event to occur for all cytokine receptor family members is the transfer of $\gamma$-phosphate of ATP to the tyrosine hydroxyl group on the receptor as well as downstream substrates (23), most notably, the STAT family of latent transcription factors (1). The STATs are recruited to the phosphorylated receptor complex through the interaction of their SH2 domain with specific phosphorylated tyrosines on the receptor. Receptor-associated STAT then becomes phosphorylated by Jak kinases and dimerizes with other phosphorylated STATs. This culminates in the nuclear translocation of dimerized STATs and ensuing transcription activation of specific target genes (1).

Jak kinases have been demonstrated to require transfer of $\gamma$-phosphates of ATP to tyrosine hydroxyl groups to increase the catalytic activity of the enzyme, a process that is believed to depend on the transphosphorylation between Jak kinases. Studies have demonstrated that phosphorylation of conserved tyrosine residues in the activation loop of Jak kinases are required for increased catalytic activity (24-27). The $\mathrm{Y}^{1007}$ site of Jak2 is also required for association with SOCS-1/JAB/SSI-1 $(28,29)$, suggesting that a key ON-OFF switch is contained within the catalytic loop. 
In addition to conserved tyrosines in the autophosphorylation loop, 12 tyrosine residues are completely conserved in the Jak kinase family, and more than 40 tyrosine residues in Jak2 are conserved between different mammalian species. Mutational analysis of the 12 conserved tyrosines individually to phenylalanine resulted in no significant effect on downstream signaling activated by Epo or IFN $\gamma$ (Feng \& Ihle unpublished). This suggests that these sites do not influence signaling or the potential effect could be compensated by additional phosphorylation sites. Thus, defining the phosphorylation pattern of Jak kinases is important for establishing the role of Jak transphosphorylation in signal transduction pathway downstream of the activated cytokine receptors. Recent studies have demonstrated that tyrosine phosphorylation events can influence the regulatory aspect of Jak kinase $(30,31)$ and as such a detailed tyrosine phosphorylation map of Jak kinases is required to address the functional consequences of additional tyrosine transphosphorylation events that occur during Jak kinase activation.

The small amounts of activated Jak kinases in a cell necessitated the establishment of alternative strategy of protein preparations to map the sites of Jak transphosphorylation. To these regards, Jak kinases overexpressed in insect cells infected with recombinant baculovirus have been demonstrated to phosphorylate STAT proteins appropriately as to confer them with specific DNA-binding activities (32). Substrate specificity also has been demonstrated by studies conducted in baculovirus-coinfected viral particles (7) suggesting that activated Jak in the baculovirus expression system responds similarly to Jak kinases activated by cytokines.

Although the functions of phosphorylation of many tyrosine kinases have been elucidated, little is understood regarding the consequence of transphosphorylation of Jak Jak2 transphosphorylation 
kinases. To begin identifying tyrosine residues that have structural/functional significance resulting from phosphorylation of tyrosine residues in Jak2, we mapped the sites of transphosphorylation in Jak2 produced in the baculovirus expression system. In this study we confirm that phosphorylation of one or both tyrosines in the $\mathrm{KEY}^{1007} \mathrm{Y}^{1008}$ transphosphorylation loop occurs. The data also indicate multiple phosphorylation sites exist in the kinase domain, as well as in other domains of Jak2.

\section{Materials and Methods}

Cell lines. Sf9 cells were maintained in Grace's insect medium supplemented with $10 \%$ fetal calf serum.

Jak2 deletion mutants and Site-directed mutagenesis: Mutational changing the conserved tyrosine residues of murine Jak2 to phenylalanine was generated by standard PCR methods as previously described $(13,24)$. The deletion constructs are a result of deletion by restriction enzyme excision as noted by the referred names or by PCR construction in D5. The mutations are defined as; AflI deletion of amino acids 253-471, BglII deletion of amino acids 523-745, EcoRI deletion of amino acids 1-295, D5 truncation of amino acids 1-756.

Immunoprecipitation and in vitro phosphorylation: Sf9 cells infected with indicated Jak2 virus were harvested and lysed (50 mM Tris-HCl, pH7.4, 0.15 M NaCl, containing 1\% Triton-X100, 10\% glycerol, 1 mM sodium orthovanadate, $1 \mathrm{mM} \mathrm{NaF,} 1$ $\mathrm{mM}$ phenylmethylsulfonyl fluoride and $10 \mathrm{ng} / \mathrm{ml}$ each of aprotinin, pepstatin and leupeptin). Jak2 proteins were immunoprecipitated from cell lysates using Jak2 antiserum (33) and proteinA-Sepharose (Sigma). In vitro kinase reactions preformed as described Jak2 transphosphorylation 
(34), briefly immune complex of Jak2 were washed in kinase buffer (10mM HEPES, ph7.4, $50 \mathrm{mM} \mathrm{NaCl}, 0.1 \mathrm{mM}$ sodium orthovanadate, $5 \mathrm{mM} \mathrm{MnCl}_{2}, 5 \mathrm{mM} \mathrm{MgCl}_{2}$ ) and mixed with $5 \mu \mathrm{Ci} / \mathrm{ml} \gamma-{ }^{32} \mathrm{P}-\mathrm{ATP}$ at $25^{\circ} \mathrm{C}$ for 30 minutes. The products of these reactions were separated by SDS-PAGE.

Phosphopeptide mapping. Analysis of phosphopeptide mapping was performed with some modification as previously described (24). Briefly, ${ }^{32} \mathrm{P}$ in vitro kinase labeled Jak2 proteins were resolved by SDS-PAGE, visualized by autoradiography and in gel digestion preformed with TPCK-treated trypsin (Sigma) and lysilendopeptidase, Lys-C (Wako). The addition of lysilendopeptidase in the digestion decreased the number of ${ }^{32} \mathrm{P}$ -labeled spots on the map in preliminary experiments by increasing complete digestion with trypsin. The proteolytic peptide mixture was separated in two dimensions on thin layer cellulose (2D-TLC) plates either in the presence or absence of unlabeled synthetic peptides as noted. ${ }^{32}$ P-labeled peptides were visualized by autoradiography and unlabeled peptide position was determined by ninhydrin staining.

Identification of tryptic peptide by reverse-phase HPLC. The ${ }^{32} \mathrm{P}$ labeled phosphopeptides of interest were extracted from thin layer cellulose plates, mixed with unlabeled synthetic phosphopeptides as noted, and separated in an Applied BioSystems HPLC system equipped with C18 reverse-phase column. Elution was accomplished with an acetonitrile gradient (0.05\% TFA from a starting $0 \%$ acetonitrile to a final $66 \%$ acetonitrile). Peptide elution was monitored by UV flow cell. Radioactivity of the eluted sample fractions was measured by Beckmann scintillation counter. 


\section{Results and Discussion}

To understand the structure/function relationship of tyrosine phosphorylation of Jak kinases the identification of Jak tyrosine phosphorylation sites is required. Studies in baculovirus produced Jak2 have been demonstrated to be activate by transphosphorylation in baculovirus and can confer DNA specific binding to purified STAT proteins (32). These studies have thereby established the efficacy of baculovirus produced Jak2 and support their use for cataloging the transphosphorylation of tyrosine.

Immunocomplexes of Jak were subjected to in vitro kinase assays in the presence of $\gamma_{-}{ }^{32} \mathrm{P}-\mathrm{ATP}$, resolved on SDS-PAGE, excised, digested with trypsin and Lys$\mathrm{C}$ and subjected to resolution on 2D TLC. Shown in Fig 1B, sixteen spots representing phosphopeptides appear. This suggests 16 as the minimum number of tyrosine containing peptides phosphorylated. Serine and threonine phosphorylation has previously been ruled out by amino acid analysis of in vitro produced Jak2 (32). ${ }^{32} \mathrm{P}$ incorporation was not evident when baculovirus produced Jak2 $\mathrm{K}^{882} \mathrm{E}$, a kinase inactive form of Jak2, was subjected to in vitro kinase assay nor was any phosphorylation noted with a antiphosphotyrosines specific western blot (data not shown), thereby establishing Jak2 as the active kinase performing transphosphorylation in the immune complexes. Each of the sixteen phosphopeptides was designated a number based on their migration pattern (Fig. $1 C)$.

It can be predicted from homology and Jak2 mutant studies that $\mathrm{Y}^{1007}$ is the tyrosine in the activation loop and phosphorylation allows access of the catalytic loop to the ATP in the ATP binding domain. Previous studies have demonstrated that the phosphorylation of JAK2 $\mathrm{Y}^{1007}$ is essential for kinase activity (24), therefore initial studies to confirm phosphorylation of $\mathrm{Y}^{1007}$ will establish the efficacy of this approach. Synthetic phosphopeptides representing the active site predicted tryptic peptide, EYYK, with the three possible phosphorylation patterns, $\mathrm{EY}^{\mathrm{P}} \mathrm{YK}, \mathrm{EYY}^{\mathrm{P}} \mathrm{K}$ and $\mathrm{EY}^{\mathrm{P}} \mathrm{Y}^{\mathrm{P}} \mathrm{K}$, were run Jak2 transphosphorylation 
on 2D-TLC with a ${ }^{32} \mathrm{P}$ in vitro labeled tryptic digested Jak2. The synthetic fragment $\mathrm{EY}^{\mathrm{P}} \mathrm{YK}$ and $\mathrm{EYY}^{\mathrm{P}} \mathrm{K}$ co-migrate with peptide designated 10 and the doubly phosphorylated $\mathrm{EY}^{\mathrm{P}} \mathrm{Y}^{\mathrm{P}} \mathrm{K}$ co-migrates with peptide 6 (Fig1D). This evidence can not however distinguish whether $\mathrm{Y}^{1008}$ can be phosphorylated without the corresponding $\mathrm{Y}^{1007}$.

To identify other phosphorylation sites in Jak2 several deletion mutations were constructed (Fig. 2A). The four deletion mutants, designated AflI, BglII, Eco RI and D5, representing kinase active Jak2 (data not shown) were subjected to mapping to allow identification of the general regions of Jak2 phosphorylation. The AflI and EcoRI mutants have the deletion in the N-terminal of Jak2, the region demonstrated to be involved with receptor association. The BgIII mutant has the JH2 domain removed and the D5 truncation is limited to $\mathrm{JH} 1$ and a small portion of the interdomain region. As expected the deletion mutants of Jak2 resulted in similar phosphopeptide maps as wt Jak2, with distinct differences attributed to the loss of tyrosine phosphorylated peptides contained within the deletion (Fig. 2 B \& C). These differences allowed the assignment of phosphotyrosines to areas within the deletion of Jak2. The absence of phosphopeptide 1 and 9 in the AflI mutant suggests these phosphopeptides are contained within amino acids 253-471. The absence of phosphopeptides 1 and 12 in the EcoRI deletion indicates that these phosphopeptides can be assigned to regions encompassing amino acids 1-295. Likewise the BglII mutant suggests that phosphopeptide 14 and 16 are contained in amino acids 523-745. Spots 3,4,5,6,8,10,11,and 15 presence in the D5 truncation, which includes the EYYK phosphorylated peptides (spots 6 and 10), demonstrates that these arise from the kinase domain.. Unexpectedly, the D5 truncation showed the loss of spots 2, 7 and 13, which could not be assigned to other Jak2 areas. The deletion mutants have in common the kinase domain and an additional region, amino acids 471-523. A single tyrosine resides in this region, which may give rise to spots 2,7 or 13 . Alternatively Jak2 transphosphorylation 
spots 2, 7 and 13 represent more that a single phosphopeptide which arise from two or more areas represented in the deletion mutants. Also likely is that spots 2, 7 and 13 contain tyrosines were the structural changes impose by the deletions cause inaccessibility of the kinase domain to the substrate tyrosine. The schematic in figure 2A assigns phosphopeptides to protein sequences on the map. The results suggest that of the sixteen phosphopeptides, six appeared to result from tyrosines in JH2-JH7, eight arise from the kinase domain and three can not be directly assigned to a region of Jak2, but appear to arise from JH2-JH7 by their absence in JH1.

To establish if conserved tyrosine phosphorylation has functional or structural significance in Jak2 tyrosines were individually replaced by phenylalanine and transfected into the Jak2 kinase deficient fibroblast cell line, $\gamma 2$ (35). With the exception of Jak2 $\mathrm{Y}^{1007} \mathrm{~F}$, no mutants were found to alter assayable signaling pathways initiated by IFN $\gamma$ or erythropoietin (Jian \& Ihle unpublished data and (13)) in the $\gamma 2$ fibroblast line. Baculovirus produced tyrosine mutants proteins were shown to have the in vitro kinase activity similar to wild-type Jak2, with the expected exception of $\mathrm{Y}^{1007}$ (data not shown). Furthermore the mutants showed similar phosphopeptide pattern compared with that of the wild type Jak2 (Fig. 2). In some cases overexposure of the 2d-TLC was necessary to visualize all the spots which incorporated ${ }^{32} \mathrm{P}$. These results surprisingly suggested that none of the tyrosine residues represented in the point mutations contribute to the phosphopeptide map. More likely the assignment of sixteen phosphopeptides does not represent the full complement of possible phosphopeptides; co-migrationing phosphopeptides could mask the loss of a peptide, adding complexity to the phosphopeptide map. The possibility of co-migrating phosphopeptides indeed makes the assignment of tyrosine phosphorylated residues a daunting task.

To deconvolute the complex pattern of Jak2 transphosphorylation an approach to match the mobility of synthetic phosphoproteins on 2D-TLC with those of the wt Jak2 Jak2 transphosphorylation 
was undertaken. Peptides that contained each of the conserved tyrosines and span the predicted trypsin sites were synthesized (Fig 3A). The synthetic peptides were in vitro phosphorylated in the presence of immune complexes of insect-derived Jak2 and separated on 17-27\% SDS-PAGE (Fig. 3B), following by in-gel tryptic digestion and resolved on 2D-TLC in the presence of synthetic phosphopeptides representing the corresponding tryptic peptide. All peptides were demonstrated to be substrate for Jak2 by ${ }^{32} \mathrm{P}$ incorporation (data not shown). Resolution on 2D-TLC allowed the assignment of each peptide to its corresponding phosphopeptide from in vitro ${ }^{32} \mathrm{P}$ transphosphorylated Jak2. The results shown in figure 3B depict the pattern of the wild type tryptic map with the mobility of each of the 32P labeled tryptic peptide and the corresponding synthetic phosphopeptide. Not shown are the patterns of synthetic peptides that did not co migrate with tryptic peptides of wtJak2. Evident is that all conserved phosphorylated tyrosines do not migrate with tryptic peptides that arise from the wild type jak2. With the exception of spot 7 and 12 all the results obtained with the synthetic peptides are supported by the deletion mutants. Spot 7 is shown to be represented by 2 peptides in the JH2-JH7 region resulting in the appearance of the spot in AgeI, BglII and EcoRI deletions, but not in the D5 deletion. Spot 12 localized in JH1 instead of the region represented by the EcoRI deletion could also result from multiple peptides, but it's absent in the D5 deletion (Fig 2 B \& C) is puzzling.

Multiple peptide migrating as a single spot was seen in peptides containing $\mathrm{Y}^{931} \mathrm{Y}^{934}$ or $\mathrm{Y}^{813}$ which co-migrated with spot 3, peptide containing $\mathrm{Y}^{119}$ or $\mathrm{Y}^{637}$ comigrated with spot 7, and peptide containing $\mathrm{Y}^{940}$ or $\mathrm{Y}^{966}$ or $\mathrm{Y}^{790}$ co-migrated with spot 8. To determine if one or more tryptic peptides are represented by a single spot synthetic phosphopeptides were analyzed by HPLC with ${ }^{32} \mathrm{P}$ labeled tryptic digested wt Jak2 spots that were excised from 2D-TLC plates. Separate HPLC runs of the synthetic peptides mixed with the radio labeled tryptic digested wt Jak2 allowed assignment of each ${ }^{32} \mathrm{P}$ Jak2 transphosphorylation 
tryptic spot with an elution profile of the UV trace representing the synthetic phosphopeptide. These results (Fig. 4) allowed assignment of tryptic peptide containing $\mathrm{Y}^{931} \mathrm{Y}^{934}$ as spot 3, tryptic peptides containg $\mathrm{Y}^{119}$ or $\mathrm{Y}^{637}$ as spot 7 and tryptic peptides containing $\mathrm{Y}^{940}$ or $\mathrm{Y}^{966}$ as spot 8 phosphopeptide from wt jak2. Likewise peptides $\mathrm{Y}^{790}$, which migrates with spot 8 and $Y^{813}$, which migrates with spot 3, can be concluded to not be transphosphorylated. Being that multiple peptides co-migrate in the three dimensions, this assay suggests the complexity of Jak2 phosphorylation. The intensity of each spot could reflect the stociometry of the phosphorylation, i.e., spots such as 3, 4 and 8 could be represented by multiple tryptic fragments. Sensitive techniques such as precursor ion trap QTRAPs (36) maybe necessary to resolve the important phosphorylation patterns of the Jaks. Attempts in these regards are being initiated to support and expand on this study.

Shown in Fig. 5 is summary of the results identifying the peptides that are transphosphorylated. Our analysis has resulted in the assignment of several transphosphorylation sites located throughout Jak2. The predominate sites of transhosphorylation appear to be proximal to the kinase domain. It is evident that transphosphorylation of Jak2 is complex and the influence of receptor association or other associated proteins have not been addressed. These studies carefully examined tyrosines that are conserved between all family members and demonstrate that these tyrosines residues are not all targets of post-translational modification (boxed peptides in Fig.5). Recent studies support that the Jak family conserved $\mathrm{Y}^{570}$ is indeed transphosphorylated, but these recent studies also indicate that a tyrosine that is uniquely conserved in Jak2 is also phosphorylated. Our studies have not targeted the identification of all the phosphorylated tyrosines apparent from the 2D TLC. Spot 12 result from the 2DTLC indicates that it is contained in the aminus termini, consistent with it being tyrosine recently identified as transphosphorylated, $\mathrm{Y}^{221}(30,31)$, but spot 12 migration with $\mathrm{Y}^{918}$ indicates that spot 12 is represented by two distinct phosphopeptides. The lack Jak2 transphosphorylation 
of appearance of spot 12 in the D5 mutant additionally suggest that structure of the kinase has an influence on transphosphorylation.

Structural modeling of Jak2 suggest that many of the peptides demonstrated to be phosphorylated in this study would be accessible to solvent others are predicted to be sterically hindered $(37,38)$. It can not be ruled out that accessibility of tyrosines would change depending on post translational modifications, such as phosphorylation. Likewise the exchange of phosphates during in vitro kinase reaction maybe influenced by structural restrictions imposed after initial transphosphorylation. Structural data will have to be obtained of activated and inactivated Jak2 to detail what structural changes will be influenced by phosphorylation. Consideration must also be made to the in vivo environment of the kinase, such as association to receptor and hetero- or homotransphosphorylation. Regardless the conserved tyrosines that are not phosphorylated are likely to be important in maintaining tertiary structure in unphosphorylated states. Future studies will address other phosphopeptides of Jak2 in a manner similar to these studies with the addition of exploiting the use of the emerging technology of mass spectrometry. The successful completion Jak2 mapping will allow a picture of complete Jak2 signaling and will put us in a position to address the different mechanisms by which the Jak2 tyrosines affect various second message cascades or regulation. 


\section{Acknowledgements}

The authors would like to thank all members of Jim Ihle’s lab (past and present), the Department of Biochemistry and the Hartwell Center for Bioinformatics and Biotechnology at St Jude Children’s Research Hospital who assisted in advice or reagents during this study.

Jak2 transphosphorylation 


\section{References}

1 J. E. Darnell, Jr., I. M. Kerr, I. M., G. R. Stark, 1994. Jak-STAT pathways and transcriptional activation in response to IFNs and other extracellular signaling proteins. Science 264 (1994) 1415-1421.

2 T. Kisseleva, S. Bhattacharya, J. Braunstein, C. W. Schindler, Signaling through the JAK/STAT pathway, recent advances and future challenges. Gene 285 (2002) $1-24$.

3 W. J. Leonard, J. J. O'Shea, 1998. Jaks and STATs: biological implications. Annu Rev. Immunol. 16 (1998) 293-322.

4 R. Binari, N. Perrimon, Stripe-specific regulation of pair-rule genes by hopscotch, a putative Jak family tyrosine kinase in Drosophila. Genes Dev. 8 (1994) 300-312.

5 P. Saharinen, K. Takaluoma, O. Silvennoinen, Regulation of the Jak2 tyrosine kinase by its pseudokinase domain. Mol. Cell. Biol. 20 (2000) 3387-3395.

6 P. Saharinen, O. Silvennoinen, The pseudokinase domain is required for suppression of basal activity of Jak2 and Jak3 tyrosine kinases and for cytokineinducible activation of signal transduction. J. Biol. Chem. 277 (2002) 4795447963.

7 B. A. Witthuhn, M. D. Williams, H. Kerawalla, Differential substrate recognition capabilities of Janus family protein tyrosine kinases within the interleukin 2 receptor (IL2R) system. Leuk. Lymphoma 32 (1999) 289.

8 M. Chen, A. Cheng, F. Candotti, Y. J. Zhou, A. Hymel, A. Fasth, L. D. Notarangelo, J. J. O'Shea, Complex effects of naturally occurring mutations in the 
JAK3 pseudokinase domain: evidence for interactions between the kinase and pseudokinase domains. Mol. Cell. Biol. 20 (2000) 947-956.

9 D. A. Harrison, R. Binari, T. S. Nahreini, M. Gilman, N. Perrimon, 1995. Activation of a Drosophila Janus kinase (JAK) causes hematopoietic neoplasia and developmental defects. EMBO J. 14 (1995) 2857-2865.

10 M. H. Shaw, V. Boyartchuk, S. Wong, M. Karaghiosoff, J. Ragimbeau, S. Pellegrini, M. Muller, W. F. Dietrich, G. S. Yap, A natural mutation in the Tyk2 pseudokinase domain underlies altered susceptibility of B10.Q/J mice to infection and autoimmunity. Proc. Natl. Acad. Sci. U S A 100 (2003) 11594-11599.

11 L. Velazquez, K. E. Mogensen, G. Barbieri, M. Fellous, G. Uze, S Pellegrini, Distinct domains of the protein tyrosine kinase tyk2 required for binding of interferon- $\alpha / \beta$ and for signal transduction. J. Biol. Chem. 270 (1995) 3327-3334.

12 D. Kampa, J. Burnside, Computational and functional analysis of the putative SH2 domain in Janus Kinases. Biochem. Biophys. Res. Commun. 278 (2000) 175.

13 F. Kohlhuber, N. C. Rogers, D. Watling, J. Feng, D. Guschin, J. Briscoe, B. A. Witthuhn, S. V. Kotenko, S. Pestka, G. R. Stark, J. N. Ihle, I. M. Kerr, 1997. A JAK1/JAK2 chimera can sustain alpha and gamma interferon responses. Mol. Cell. Biol. 17 (1997) 695-706.

14 A. H. Chishti, A. C. Kim, S. M. Marfatia, M. Lutchman, M. Hanspal, H. Jindal, S. C. Liu, P. S. Low, G. A. Rouleau, N. Mohandas, J. A. Chasis, J. G. Conboy, P. Gascard, Y. Takakuwa, S. C. Huang, E. J. Benz, Jr., A. Bretscher, R. G. Fehon, J. F. Gusella, V. Ramesh, F. Solomon, V. T. Marchesi, S. Tsukita, K. B. Hoover, et 
al., The FERM domain: a unique module involved in the linkage of cytoplasmic proteins to the membrane. Trends Biochem. Sci. 23 (1998) 281-282.

15 J. A. Girault, G. Labesse, J. P. Mornon, I. Callebaut, 1998. Janus kinases and focal adhesion kinases play in the 4.1 band: a superfamily of band 4.1 domains important for cell structure and signal transduction. Mol. Med. 4 (1998) 751-769.

16 J. A. Girault, G. Labesse, J. P. Mornon, I. Callebaut, The N-termini of FAK and JAKs contain divergent band 4.1 domains. Trends Biochem. Sci . 24 (1999) 54-57.

17 N. A. Cacalano, T. S. Migone, F. Bazan, E. P. Hanson, M. Chen, F. Candotti, J. J. O'Shea, J. A. Johnston, Autosomal SCID caused by a point mutation in the Nterminus of Jak3: mapping of the Jak3-receptor interaction domain. EMBO J. 18 (1999) 1549-1558.

18 R. A. Anderson, V. T. Marchesi, Regulation of the association of membrane skeletal protein 4.1 with glycophorin by a polyphosphoinositide. Nature 318 (1985) 295-298.

19 T. L. Leto, V. T. Marchesi, A structural model of human erythrocyte protein 4.1. J. Biol. Chem. 259 (1984) 4603-4608.

20 A. Usacheva, S. Kotenko, M. M. Witte, O. R. Colamonici, Two distinct domains within the N-terminal region of Janus kinase 1 interact with cytokine receptors. J. Immunol. 169 (2002) 1302-1308.

21 C. M. Hilkens, H. Is'harc, B. F. Lillemeier, B. Strobl, P. A. Bates, P. A., I. Behrmann, I. M. Kerr, A region encompassing the FERM domain of Jak1 is necessary for binding to the cytokine receptor gp130. FEBS Lett. 505 (2001) 8791.

Jak2 transphosphorylation 
22 K. He, X. Wang, J. Jiang, R. Guan, K. E. Bernstein, P. P. Sayeski, G. J. Frank, JAK2 Determinants for GH Receptor Association, Surface Assembly, and Signaling. Mol. Endocrinol. 17 (2003) 2211-2227.

23 D. M. Wojchowski, R. C. Gregory, C. P. Miller, A. K. Pandit, T. J. and Pircher, 1999. Signal transduction in the erythropoietin receptor system. Exp. Cell. Res. 253 (1999) 143-156.

24 J. Feng, B. A. Witthuhn, T. Matsuda, F. Kohlhuber, I. M. Kerr, J. N. Ihle, Activation of Jak2 catalytic activity requires phosphorylation of Y1007 in the kinase activation loop. Mol. Cell. Biol. 17 (1997) 2497-2501.

25 K. D. Liu, S. L. Gaffen, M. A. Goldsmith, W. C. Greene, Janus kinases in interleukin-2-mediated signaling: JAK1 and JAK3 are differentially regulated by tyrosine phosphorylation. Curr. Biol. 7 (1997) 817-826.

26 R. Wang, P. R. Griffin, E. C. Small, J. E. Thompson, Mechanism of Janus kinase 3-catalyzed phosphorylation of a Janus kinase 1 activation loop peptide. Arch Biochem Biophys 410 (2003) 7-15.

27 Y. J. Zhou, E. P. Hanson, Y. Q. Chen, K. Magnuson, M. Chen, P. G. Swann, R. L. Wange, P. S. Changelian, J. J. O'Shea, Distinct tyrosine phosphorylation sites in JAK3 kinase domain positively and negatively regulate its enzymatic activity. Proc. Natl. Acad. Sci. U S A 94 (1997) 13850-13855.

28 D. Ungureanu, P. Saharinen, I. Junttila, D. J. Hilton, O. Silvennoinen, Regulation of Jak2 through the ubiquitin-proteasome pathway involves phosphorylation of Jak2 on Y1007 and interaction with SOCS-1. Mol. Cell. Biol. 22 (2002) 33163326.

Jak2 transphosphorylation 
29 H. Yasukawa, H. Misawa, H. Sakamoto, M. Masuhara, A. Sasaki, T. Wakioka, S. Ohtsuka, T. Imaizumi, T. Matsuda, J. N. Ihle, A. Yoshimura, The JAK-binding protein JAB inhibits Janus tyrosine kinase activity through binding in the activation loop. EMBO J. 18 (1999) 1309.

30 E. P. Feener, F. Rosario, S. L. Dunn, Z. Stancheva, M. G. Myers, Jr., Tyrosine phosphorylation of Jak2 in the JH2 domain inhibits cytokine signaling. Mol. Cell. Biol. 24 (2004) 4968-4978.

31 Argetsinger, L. S., Kouadio, J. L., Steen, H., Stensballe, A., Jensen, O. N., and Carter-Su, C. 2004. Autophosphorylation of JAK2 on tyrosines 221 and 570 regulates its activity. Mol. Cell. Biol. 24:4955-4967.

32 F. W. Quelle, W. Thierfelder, B. A. Witthuhn, B. Tang, S. Cohen, J. N. Ihle, 1995. Phosphorylation and activation of the DNA binding activity of purified Stat1 by the Janus protein-tyrosine kinases and the epidermal growth factor receptor. J. Biol. Chem. 270 (1995) 20775-20780.

33 O. Silvennoinen, B. A. Witthuhn, F. W. Quelle, J. L. Cleveland, T. Yi, J. N. Ihle, 1993. Structure of the murine Jak2 protein-tyrosine kinase and its role in interleukin 3 signal transduction. Proc. Natl. Acad. Sci. U S A 90 (1993) 84298433.

34 B. A. Witthuhn, F. W. Quelle, O. Silvennoinen, T. Yi, B.Tang, O. Miura, J. N. Ihle, JAK2 associates with the erythropoietin receptor and is tyrosine phosphorylated and activated following stimulation with erythropoietin. Cell 74 (1993) 227-236.

Jak2 transphosphorylation 
35 D. Watling, D. Guschin, M. Muller, O. Silvennoinen, B. A. Witthuhn, F. W. Quelle, N. C. Rogers, C. Schindler, G. R. Stark, J. N. Ihle, et al. , Complementation by the protein tyrosine kinase JAK2 of a mutant cell line defective in the interferon-gamma signal transduction pathway. Nature 366 (1993) 166-170.

36 H. Steen, B. Kuster, M. Fernandez, A. Pandey, M. Mann, Detection of tyrosine phosphorylated peptides by precursor ion scanning quadrupole TOF mass spectrometry in positive ion mode. Anal Chem 73 (2001) 1440-1448.

37 F. Giordanetto, R. T. Kroemer, Prediction of the structure of human Janus kinase 2 (JAK2) comprising JAK homology domains 1 through 7. Protein Eng. 15 (2002) 727-737.

38 K. Lindauer, T. Loerting, K. R. Liedl, R. T. Kroemer, Prediction of the structure of human Janus kinase 2 (JAK2) comprising the two carboxy-terminal domains reveals a mechanism for autoregulation. Protein Eng. 14 (2001) 27-37.

Jak2 transphosphorylation 


\section{Figure Legends}

Figure 1. Jak2 transphosphorylation. A. Schematic view of Jak2 showing the designated Jak homology domains and the interdomain regions (crosshatched). The tyrosines are designated by their single letter. Tyrosine conserved throughout the Jak family represented by $\mathbb{Y}$ and those limited to Jak2 are represented by Y. B.) baculovirus produced Jak2 was immunoprecipitated, subjected to in vitro kinase reaction and resolved by SDS-PAGE. The excised band containing Jak2 were treated by in-gel tryptic digestion, peptides extracted and resulting lyophilized product resolved by 2D-TLC and visualized by autoradiograph. C.) Schematic view of the assignment of the tryptic peptides resolved on 2D-TLC. D.) Schematic view of the peptides contained within the autophosphorylation site determined by synthetic peptides representing EYYK singly or doubly phosphorylated were resolved on 2D TLC, visualize with ninhydrin and assigned to migration pattern of wtJak2 phosphopeptide with identical mobility.

Figure 2. Mutant Jak2 transphosphorylation. A.) Schematic representation of Jak2 and the various deletion or truncation mutations. The number assignment designated on the schematic are the peptide assignment determined by the loss of the tryptic peptide as shown in Figure 2. B and C. B.) The 2D TLC resolved tryptic peptides obtained from baculovirus produced protein as described in fig 1 . C. Schematic view of each of the deletion or truncated mutants showing the migration pattern in relationship to the migration of phosphopeptide migration of the wild type.

Figure 3. Jak2 synthetic peptide phosphorylation, tryptic digestion and 2DTLC resolution. A. Peptides synthesized with flanking trypsin recognition site. The predicted trypsin digest phosphopeptides products were also synthesized and designates by the corresponding tyrosine number from the sequence (33). B.) Synthetic peptides (designated by letter assignment in Fig 3A) were ${ }^{32} \mathrm{P}$ labeled with wtJak2 immunoprecipitated complexes, separated on SDS-PAGE 12-25\% gradient gel and Jak2 transphosphorylation 
subjected to in gel tryptic digestion. Samples were resolved on 2D-TLC with the predicted tryptic phosphopeptide. Dashed circle represent the migration of ninhydrin stained phosphopeptide and ${ }^{32} \mathrm{P}$ labeled protein is visualized by autoradiograph. Migration is determined in relationship to wt Jak2.

Figure 4. HPLC correlation of elution pattern of synthetic phosphopeptide with the in vitro kinase 32P labeled tryptic synthetic peptide. A. Synthetic phosphopeptides representing the tryptic peptides with identical 2D TLC migrations

patterns were resolved in the presence of ${ }^{32} \mathrm{P}$ in vitro labeled tryptic digested synthetic peptides. The elution pattern of the unlabeled phosphopeptide was monitored by absorption and the collected fractions were monitored by radioactivity in a Beckman scintillation counter. Arrows designate the elution profile determined from flow cell uv tracing of the synthetic peptide.

Figure 5: Schematic view of the phosphopeptides determined in this study. Boxed below are the phosphopeptides which results of this study determined as not phosphorylated in in vitro kinase assay.

Jak2 transphosphorylation 
A

\begin{tabular}{|c|c|c|c|c|c|c|c|c|}
\hline JH7 & JH6 & JH5 & JH4 & JH3 & & JH & & JH1 \\
\hline ry mrrry & $\mathrm{Y} \mathrm{mrr}_{\mathrm{f}} \mathrm{Y}$ & & $\mathbf{Y} \quad / \mathbf{Y} / \mathbf{Y}$ & r wry & 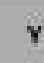 & 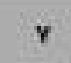 & \begin{tabular}{ll|l}
$\mathrm{v}$ & $\mathrm{N}$ & $\mathrm{r}$
\end{tabular} & Y Y wrYY Y YYYY YYY \\
\hline
\end{tabular}

$7:$ Conserved tyrosine residue

B
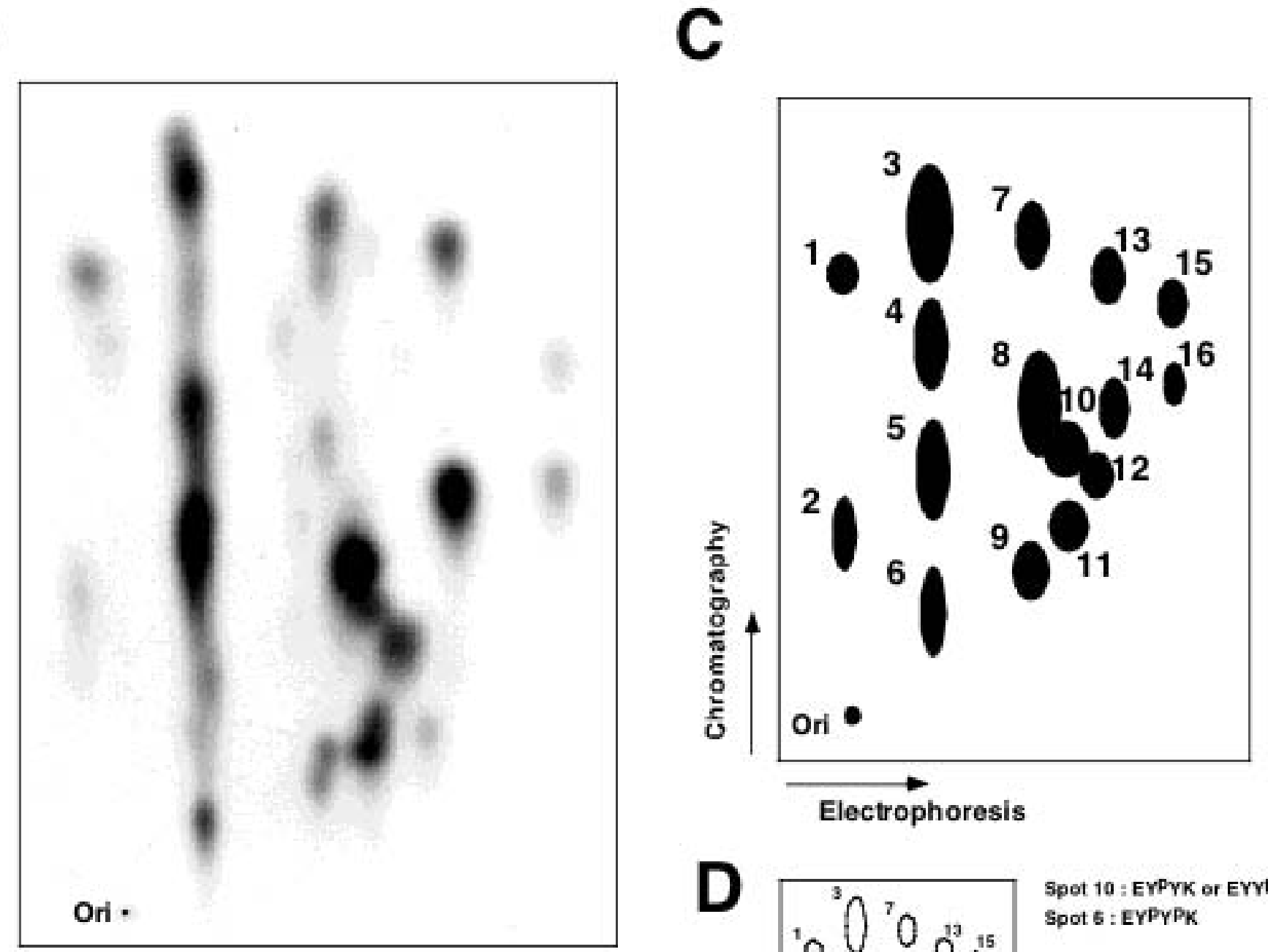

D

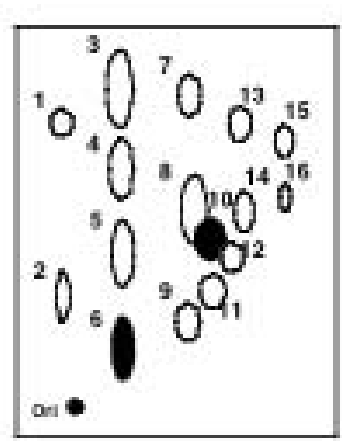

Spot 10 : EYPYK or EYYPK Spot 6 : EYPYPK 
A

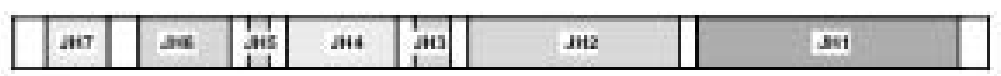

Jak2 WT

Jak2 / Afl I

Jak2 / Bgl II

Jak2 / Eco RI

Jak2 / D5

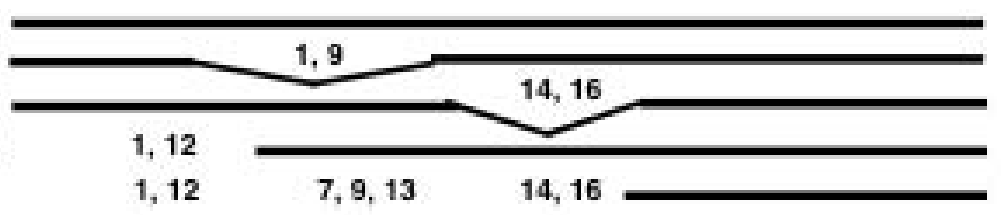

B Afl I

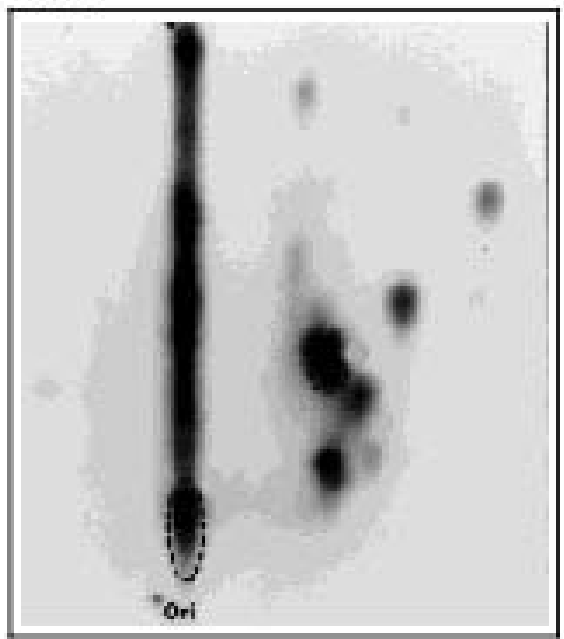

Bgl II

\section{EcoRI}
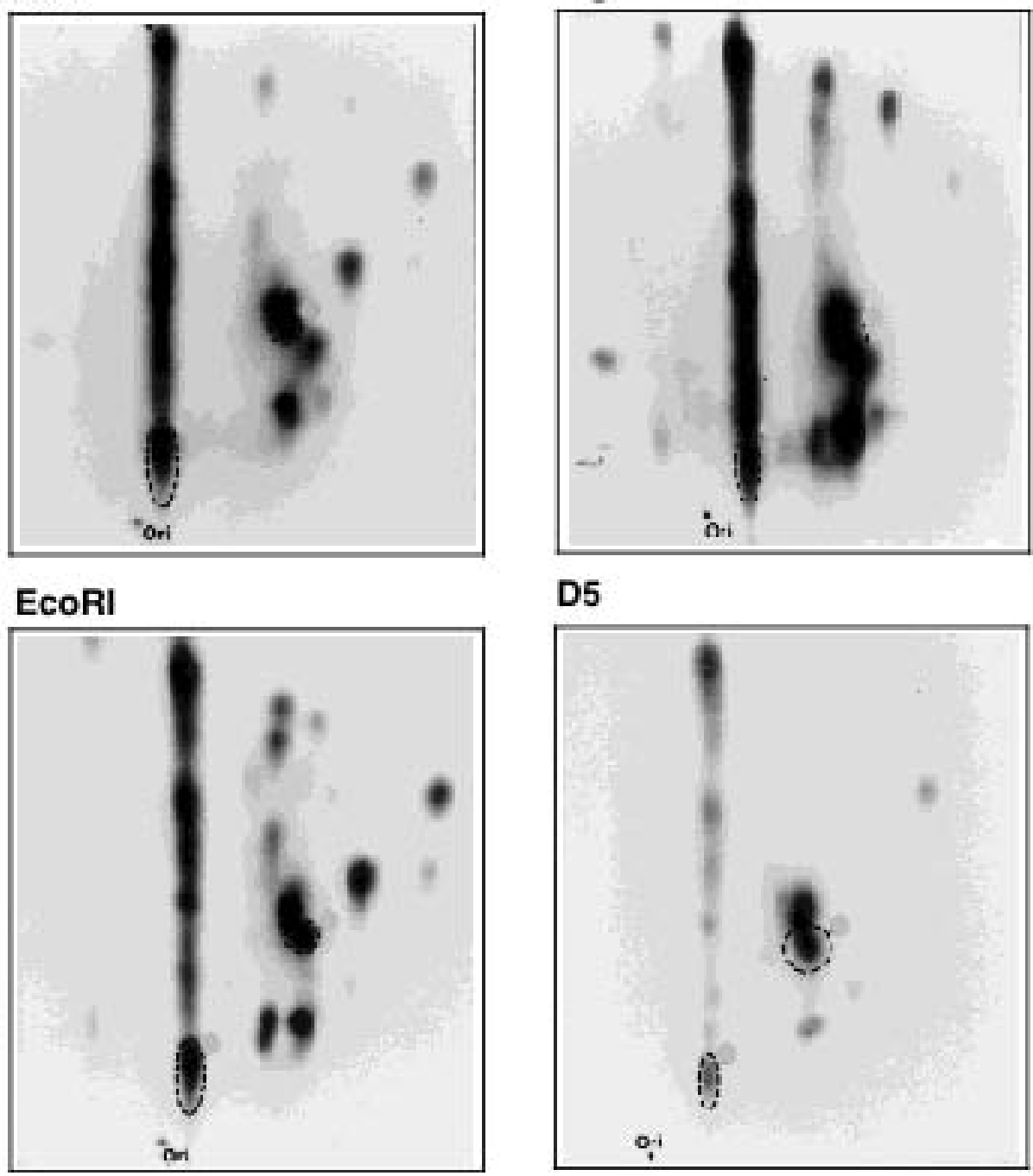

D5

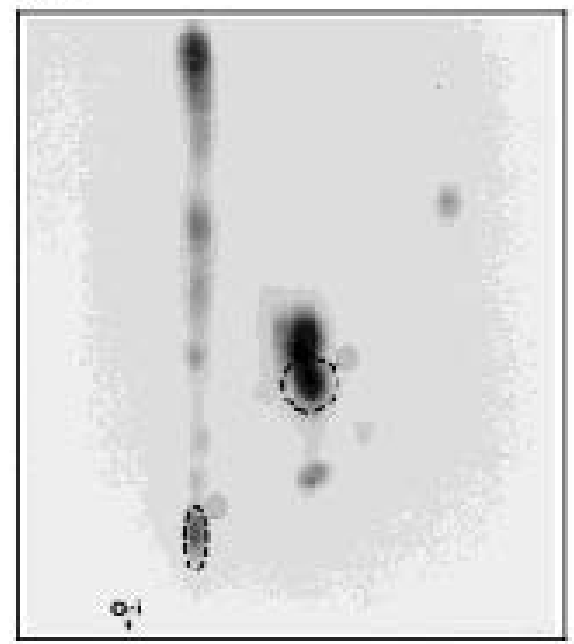

C
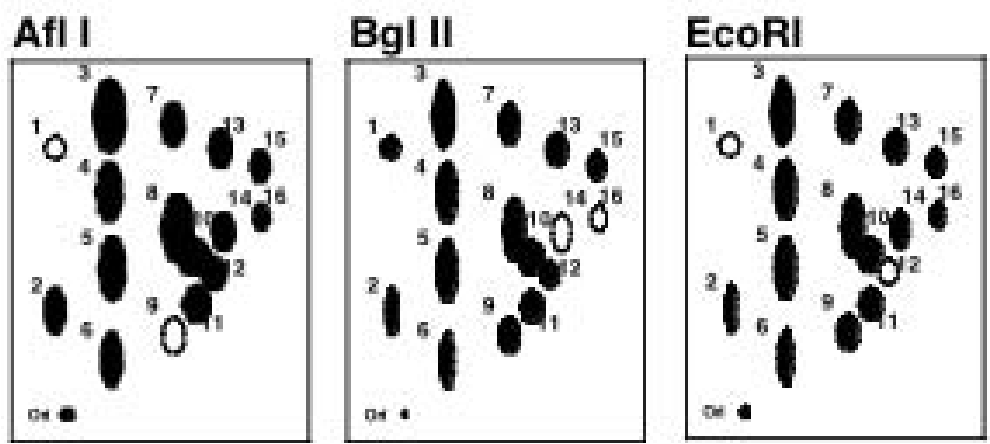

D5

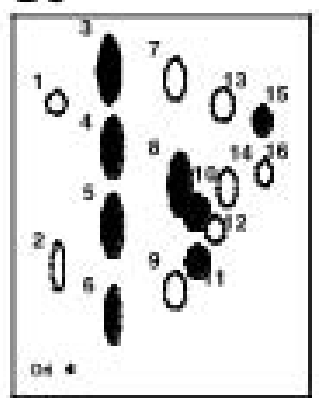

Fig. 2 
Synthetic peptide

\begin{tabular}{|c|c|}
\hline $\mathrm{N}$-terminal domain & A. Y119:RIRFYFPHWYCSGSSR \\
\hline & B. Y254:LKYLINLETLOSAFYTEOFEVKE \\
\hline & C. Y372/Y382:EALSFVSLIDGYYRLTADAHHYLCK \\
\hline & D. Y423:LKKAGNOTGLYVLRCS \\
\hline Kinase-like domain & E. Y570:GVRREVGDYGQLHKTEVLLK \\
\hline & F. Y590:AHRNYSESFFEAASMMSQLSHK \\
\hline & G. Y613:HLVLNYGVCVCGEENILVQEFVK \\
\hline & H. Y637:VQEFVKFGSLDTYLKKNK \\
\hline & L. Y766:SQRKLOFYEDKHQL \\
\hline & J. Y790:WTELANLINNCMDYEPDFR \\
\hline & K. Y813:DLNSLFTPDYELLTENDMLPNMR \\
\hline Kinase domain & L. Y868:VEMCRYDPLODNTGEVVAVK \\
\hline & M. Y913/Y918:LQHDNIVKYKGVCYSAGR \\
\hline & N. Y931/Y934/Y940:GRRNLRLIMEYLPYGSLRDYLOK \\
\hline & O. Y956/Y966:KKLLQYTSOICKGMEYLGTK \\
\hline & P. Y966/Y972:QICKGMEYLGTKRYIHR \\
\hline & Q. Y1007/Y1008:DFGLTKVLPQDKEYYKVKEPGESPIF \\
\hline & R. Y1021:EPGESPIFWYYAPESLTESK \\
\hline & S. Y1045/Y1050:SFGVVLYELFTYIEKSKS \\
\hline & T. Y1099:PEGCPDEIYVIMTECWNNNVSQR \\
\hline
\end{tabular}

Predicted tryptic/Lys-C peptide

FYFPHWYCSGSSR

YLINLETLOSAFYTEOFEVK

EALSFVSLIDGYYR / LTADAHHYLCK

AGNOTGLYVLR

EVGDYGQLHK

NYSESFFEAASMMSQLSHK

HLVLNYGVCVCGEENILVOEFVK

FGSLDTYLK

LQFYEDK

WTELANLINNCMDYEPDFR

DLNSLFTPDYELLTENDMLPNMR

YDPLODNTGEVVAVK

YK/GVCYSAGR

LIMEYLPYGSLR / DYLOK

LLOYTSOICK / GMEYLGTK

GMEYLGTKR / YIHR

EYYK

EPGESPIFWYYAPESLTESK

SFGVVLYELFTYIEKSK

PEGCPDEIYVIMTECWNNNVSQR

\section{Fig. 3}




\section{B}

A: Spot $7-Y^{119}$

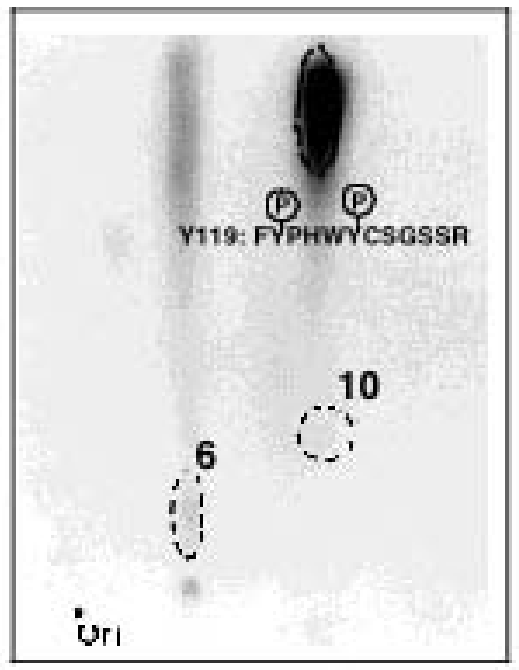

B: Spot $14 \& 16-Y^{570}$

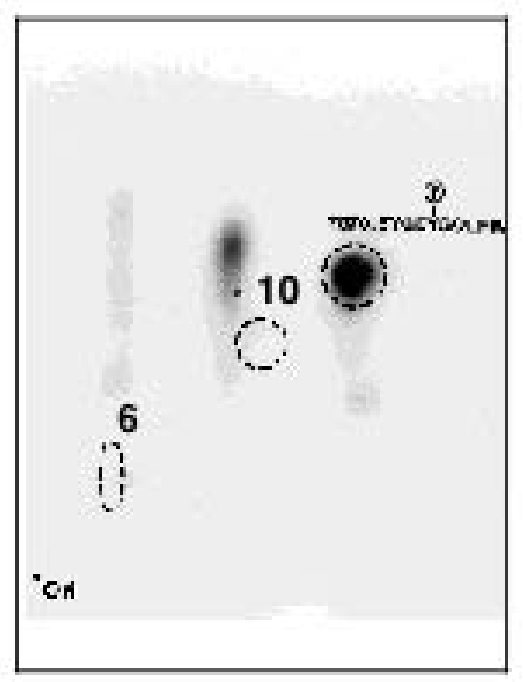

C: Spot $7-Y^{637}$

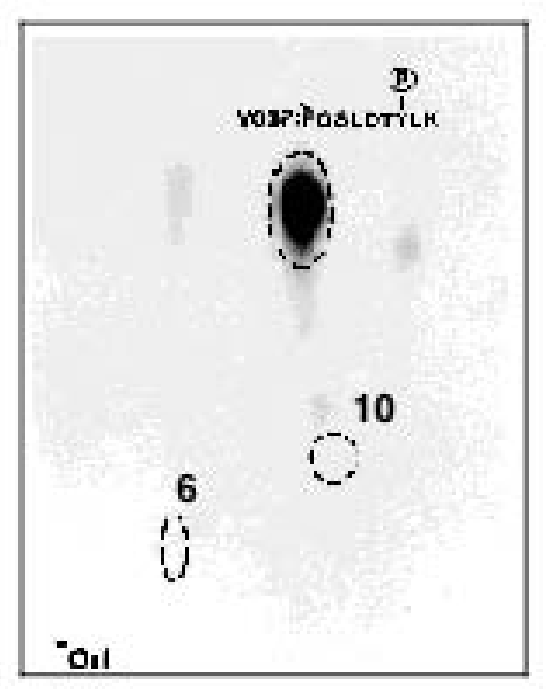

D: Spot 12-Y918

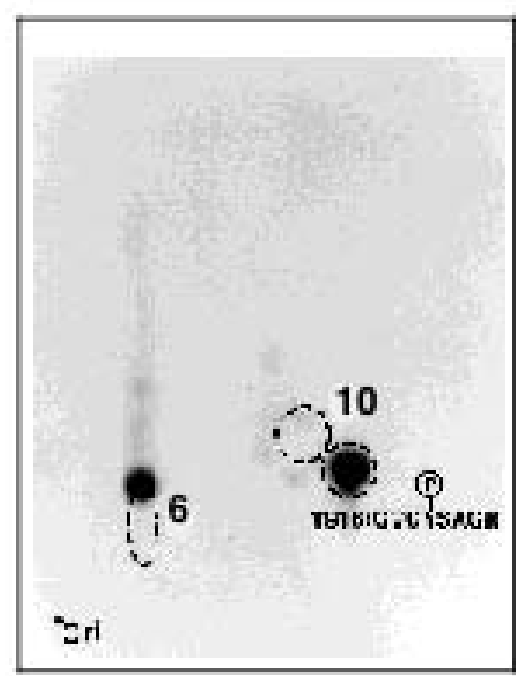



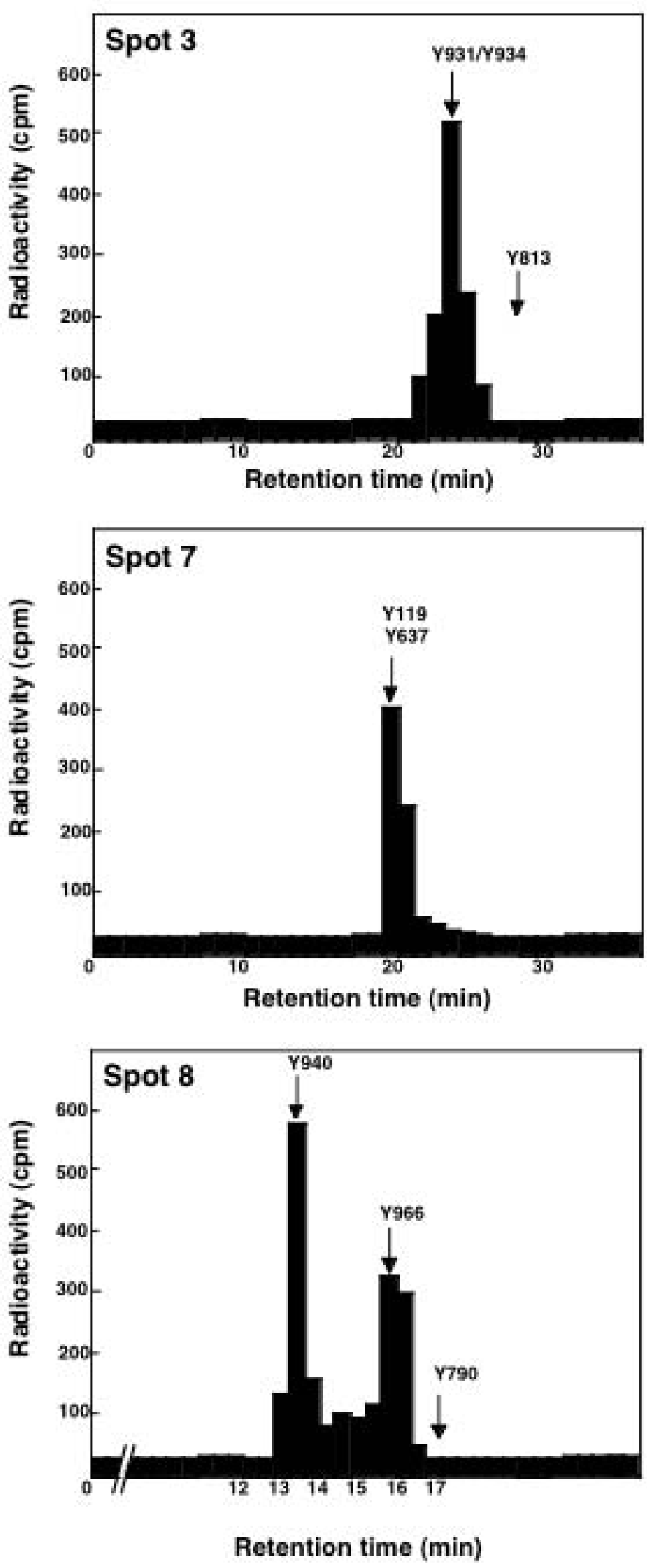

Fig. 4 


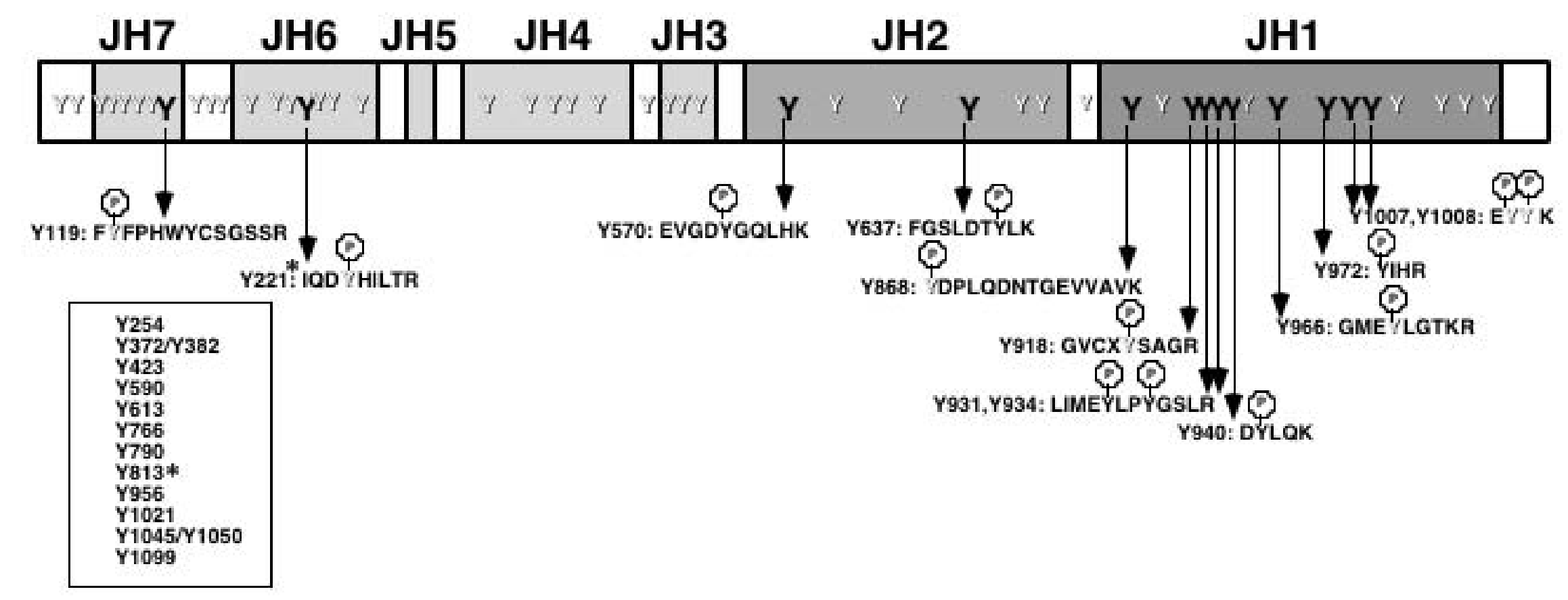

Fig. 5 